Emergency Medicine Residency Training Program, York, PA, USA, ${ }^{3}$ Department of Emergency Medicine, University of Alabama at Birmingham, Birmingham, AL, USA

Objective.-To create a longitudinal wilderness medicine curriculum or track that can be incorporated into an emergency medicine (EM) residency program.

Methods.-Interest-specific tracks are becoming increasingly popular in EM training. We chose this model to develop our curriculum specific to wilderness medicine (WM). This curriculum may be incorporated into an EM training program. This training would facilitate transition to a WM fellowship or other career in the field for the interested resident. Outlined is a 3-year longitudinal course of study that includes a core didactic curriculum and a plan for graduated levels of responsibility. The core content is specifically related to the required EM core content for residency training. The track enhances the WM specific core content for the EM residents as a whole, in addition to providing each WM track resident with additional focused WM didactics, skills, and teaching opportunities. Finally, the track allows the resident to align his or her research, didactic, and administrative project requirements of the EM program within the field of WM study. The WM track curriculum is easily modified to fit the particular needs of individual residency programs.

Results.-We present a model core content curriculum for a WM track for training within an EM training program.

Conclusions.-This WM curriculum would give residencies a framework that can be used to foster learning for the residents interested WM. It enhances the coverage of wilderness core content education for all of the EM residents in the program. It provides wilderness- and environmental-specific education and experience for interested residents, allowing them to focus on an area of study, enhancing their CVs at graduation. Finally, given the popularity of WM, the presence of a WM track may potentially improve recruitment for the residency program.

\section{Prospective Double-Blinded Randomized Field-Based Clinical Trial of Metoclopramide and Ibuprofen for the Treatment of Acute Mountain Sickness}

Hillary R. Irons, $\mathrm{MD}, \mathrm{PhD}^{1}$; Renee N. Salas, $\mathrm{MD}^{1}$; Salman

F. Bhai, $\mathrm{MD}^{2}$; N. Stuart Harris, MD

${ }^{1}$ Massachusetts General Hospital, Boston, MA, USA, ${ }^{2}$ Brigham and

Women's Hospital, Boston, MA, USA

Introduction.-Acute Mountain Sickness (AMS) afflicts 50\% to $80 \%$ of high-altitude travelers, and can be debilitating. AMS is defined as headache with nausea/vomiting, fatigue/weakness, dizziness/lightheadedness, and/or difficulty sleeping because of rapid ascent. Becaue patients with AMS often are nauseated, metoclopramide may be a solution, as it treats nausea and migraine headaches. Studies also suggest ibuprofen as effective AMS therapy.

Objective.-Assess the effectiveness of metoclopramide compared with ibuprofen for AMS treatment.

Methods.-We performed a prospective, double-blinded, randomized, field-based clinical trial of $10 \mathrm{mg}$ metoclopramide vs $400 \mathrm{mg}$ ibuprofen for AMS treatment. Enrollment was March to May 2013 and March to April 2015 along the approach to Mount Everest in Pheriche $(4280 \mathrm{~m})$ and Dingboche (4358 m), Nepal. Eligibility criteria were $\geq 1000$ vertical feet gain over 24 hours with a headache and one other AMS symptom. Patients with severe AMS, high altitude cerebral edema, or high-altitude pulmonary edema were excluded. Subjects were assessed by vital signs, Lake Louise Score (LLS), and a Visual Analog Scale for headache and nausea severity immediately prior to treatment, and then serially at 30,60, and 120 minutes afterwards. Results were analyzed by signed-rank and Wilcoxon rank-sum tests $(P$ values $<.05$ considered significant).

Results. - Analysis revealed no significant differences between metoclopramide and ibuprofen groups with respect to age, sex, initial LLS, heart rate, or oxygen saturation. Both medications significantly reduced headache severity and nausea compared with initial headache and nausea over the 2-hour study. At 120 minutes, metoclopramide additionally reduced nausea to a greater degree than ibuprofen, although both medications reduced overall AMS symptoms. When the LLS was used, there was a significant decrease in headache, dizziness, and total LLS with ibuprofen compared with metoclopramide.

Conclusion.-Both medications effectively reduced AMS symptoms, including headache and nausea. Metoclopramide additionally reduced nausea more than ibuprofen. Therefore, metoclopramide may be an effective alternative AMS treatment, especially for patients with nausea.

Funding: WMS Houston Grant (2016).

\section{Promoting Resilience Among Veterans Using Wild- erness Therapy}

Shauna Joye; Zachary Dietrich

Georgia Southern University, Statesboro, GA, USA

Introduction.-Mental health problems are common among combat veterans. Although the US government has set aside resources to treat these individuals, long wait times, stigma related to receiving treatment, and the perception of few treatment benefits remain barriers for soldiers seeking mentalhealth services. One alternative to traditional psychotherapy is wilderness therapy, which uses the natural environment to promote recovery.

Objective.-The purpose of the present research was to expand on the literature and our previous findings by measuring the mental health of 7 groups of combat veterans on long-distance (ie, 4- 6 months) hiking and paddling trails.

Methods. - Veterans completed pre-, mid-, and posthike measures aimed at gauging their mental-health functioning. We also emailed them weekly psychoeducational messages about how they might think and behave in ways to promote well-being. For 3 of the trails, we hiked with the veterans for 2 days at the beginning of their wilderness experience.

Results.-Preliminary data analyses for veterans in our wilderness therapy program vs those in a waitlist control group indicated larger gains in mental health across 11 variables for those in the treatment group. Even with a small sample size $(\mathrm{N}$ $=31)$, we found statistically significant differences between groups for anxiety $(P<$ $.001)$, phobic anxiety $(P=.04)$, and interpersonal sensitivity $(P=.03)$, and a marginally significant difference in overall mental health $(P=.06)$.

Conclusions.-For a generation of post-9/11 combat veterans who may not benefit from traditional approaches to therapy, our wilderness therapy program showed promise as a way for veterans to address issues related to experiencing traumatic events while deployed. We found significant decreases in general anxiety, anxiety related to fear or uneasiness when in new places and around crowds, and feelings of wariness or self-consciousness around others.

Funding: WMS Hackett-Auerbach Grant (2015).

\section{Ambulatory Blood Pressure Monitoring Reveals Increased Sleeping Blood Pressure in Hypertensive Individuals at High Altitude}

David S. Young, MD, $\mathrm{MS}^{1}$; Linda E. Keyes, $\mathrm{MD}^{1}$; Luke F. Mather, $\mathrm{MD}^{2}$; Charles Duke ${ }^{3}$; Nirajam Rejmi, $\mathrm{MD}^{4}$; Benoit M. Phelan, MD ${ }^{5}$; Sushil Pant, $\mathrm{MD}^{4}$; Jennifer M. Starling, $\mathrm{MD}^{1}$; Matthew K. McElwee ${ }^{6}$; Devlin Cole $^{6}$; Theodore McConnell ${ }^{7}$; Purshotam Paudel ${ }^{4}$; Douglas; Sallade ${ }^{8}$; Allison L. Sheets, MD ${ }^{9}$; David R. Twillman ${ }^{1}$; Buddha Basynat, $\mathrm{MD}^{10}$

${ }^{1}$ University of Colorado Denver School of Medicine, Aurora, CO, USA, ${ }^{2}$ University of Washington School of Medicine, Seattle, WA, USA, ${ }^{3}$ University of Tennessee Health Science Center, Memphis, TN, USA, ${ }^{4}$ Mountain Medicine Society of Nepal, Kathmandu, Nepal, ${ }^{5}$ Dalhousie University Faculty of Medicine, Halifax, NS, Canada, ${ }^{6}$ Case Western Reserve University School of Medicine, Cleveland, $\mathrm{OH}, \mathrm{USA},{ }^{7} \mathrm{McGill}$ University, Montreal, QC, Canada, ${ }^{8}$ Philadelphia College of Osteopathic Medicine, Philadelphia, PA, USA, ${ }^{9}$ Longmont United Hospital, Longmont, CO, USA, ${ }^{10}$ Oxford University Clinical Research Unit Nepal, Patan, Nepal

Introduction.-For those traveling to high altitude, it has been postulated that blood pressure (BP) changes when compared to sea level. Also at altitude, periodic breathing has been well described and is correlated to decreased sleep quality.

Objective.-Measure continuous sleeping and awake blood pressures in both normotensive and hypertensive Himalayan trekkers.

Methods.-Blood pressure was measured with Welch-Allyn 6100 24h ambulatory BP monitors (24h-ABPM) every 30 minutes while awake and every 